\title{
Theoretical Calculations of Extrinsic Parameters of Camera
}

\author{
Jiangshan Liu \\ School of North China Electric Power University, Baoding 071000, China. \\ ailrg103@qq.com
}

Keywords: P4P, extrinsic parameters, artificial identification, pose

\begin{abstract}
Monocular vision technology can be applied to calculate the tilt angle of the insulator. Camera extrinsic parameters describe the translation and rotation parameters also called pose between the world coordinate system and the camera coordinate system. Thus, determining the extrinsic parameters is a very important part of the process. We set artificial identification to determine the positional relationship to determine the extrinsic parameters. There are four artificial landmarks to determine the orientation of the camera, which is P4P problem. The method is feasible within the range of allowable error.
\end{abstract}

\section{Introduction}

With the continuous development of visual technology, it has a wide range of applications in the industrial, medical, aerospace, military and other fields. Visual technology can replace human beings to work in size detection, target tracking, robot navigation, etc. And it is more suitable for measurement with high repeatability, non-contact precision. Insulators tilt angle is an important monitoring parament whose accuracy has direct impact on the of the calculation on equivalent icing thickness of cables. Monocular vision technology to identify the inclination angle of the insulator string from an image, avoiding large labor-intensive manual testing, fatigue, low efficiency shortcomings. For the non-contact measurement insulator inclination angle provides a new way of thinking. Monocular vision technology provides a new way for the non-contact measurement of insulator tilt angle.

\section{Solving camera extrinsic parameters}

\section{Solving P4P Problem about four points in the same plane[1].}

Applicating the P4P in camera location problem. $S$ is the origin of the camera coordinate system, which is the camera optical center. $a, b, c, d$ are the projection points in the image plane of the four spatial points $A 、 B 、 C 、 D$.In case that the internal reference of camera is known, we can obtain $\angle a S b 、 \angle b S c, \angle c S d, \angle d S a$ and the unit vectors along with the directions of $S a, S b 、 S c 、 S d$ in the camera coordinate system at the same time.

The steps of recovering the coordinates of $A 、 B 、 C 、 D$ in the camera coordinate system:

(1) Decomposing the inside and outside parameters matrix of the camera to get the three-dimensional coordinate $\mathrm{w}_{\mathrm{c}}$ of the optical center $\mathrm{S}$;

(2) Calculating the unit vector $a_{i}$ between the optical center and every feature point;

(3) Calculating the distance $\mathrm{L}_{\mathrm{i}}$ between the optical center and every feature point;

(4) Determining the spatial coordinate $\mathrm{S}_{\mathrm{i}}=\mathrm{L}_{\mathrm{i}} \mathrm{a}_{\mathrm{i}}+\mathrm{W}_{\mathrm{c}}$ of every feature point;

The key to this method is to calculate the distance between the optical center and every feature point, which can be transformed into solving the problem of P4P.[2]

Make $|S A|=L_{1},|S B|=L_{2},|S C|=L_{3},|D A|=L_{D A},|A B|=L_{A B},|B C|=L_{B C},|C D|=L_{C D},|D A|=L_{D A},|A C|=L_{A C},|B D|=L_{B D}$.In $\triangle S A B$ 、 $\triangle S B C 、 \triangle S C D 、 \triangle S D A 、 \triangle S A C$, the following conclusions can be obtained by the law of cosines.

Connecting $A C, B D$, the two lines intersect at the point $E$.Also,we can get the point $e$ in the image plane. In the image plane,the physical image coordinates of $a, b, c, d$ and $e$ are known,so the length of the line $a e$ can be easily obtained. The inside camera parameters are known,by whcih 
the length of $S a$ and $S e$ can be easily obtained.In $\triangle S a e$, we can determine $\angle a S e, \angle c S e$ in accordance with the law of cosines.In $\triangle S A E 、 \triangle S C E$, applying the law of sines respectively, the following formula can be finished:

Set:

$$
\begin{array}{r}
L_{1}=\frac{\sin (\angle C S E)}{|C E|} \times \frac{|A E|}{\sin (\angle A S E)} \times L_{3} \\
m=\frac{\sin (\angle C S E)}{|C E|} \times \frac{|A E|}{\sin (\angle A S E)}
\end{array}
$$

Set:

$$
\begin{array}{r}
L_{2}=\frac{\sin (\angle D S E)}{|D E|} \times \frac{|B E|}{\sin (\angle B S E)} \times L_{4} \\
n=\frac{\sin (\angle D S E)}{|D E|} \times \frac{|B E|}{\sin (\angle B S E)}
\end{array}
$$

Taking $m, n$ into formula:

$$
\left\{\begin{array}{c}
m^{2} L_{3}{ }^{2}+n^{2} L_{4}{ }^{2}-2 m n \cos (\angle A S B) L_{3} L_{4}=L_{A B}{ }^{2} \\
L_{3}{ }^{2}+n^{2} L_{4}{ }^{2}-2 n \cos (\angle B S C) L_{3} L_{4}=L_{B C}{ }^{2} \\
L_{3}{ }^{2}+L_{4}{ }^{2}-2 \cos (\angle C S D) L_{3} L_{4}=L_{C D}{ }^{2} \\
m^{2} L_{3}{ }^{2}+L_{4}{ }^{2}-2 m \cos (\angle D S A) L_{3} L_{4}=L_{D A}{ }^{2} \\
m^{2} L_{3}{ }^{2}+L_{3}{ }^{2}-2 m \cos (\angle A S C) L_{3}{ }^{2}=L_{A C}{ }^{2} \\
n^{2} L_{4}{ }^{2}+L_{4}{ }^{2}-2 n \cos (\angle B S D) L_{4}{ }^{2}=L_{B D}{ }^{2}
\end{array}\right.
$$

The value of ${ }^{L_{3}},{ }^{L_{4}}$ can be obtained directly by the last two of formula(5).

Taking ${ }^{L_{3}}, L_{4}$ into formula(1) and formula(3),we can get the value of $L_{1}, L_{2}$.

Such as the calculation of the camera coordinate of point A. Based on the conversion relationship between image coordinates and the image coordinates,we can obtain camera coordinates.The vector $\overrightarrow{S a}$ and the vector $\overrightarrow{S A}$, the distance ${ }^{|S a|}$ and the distance ${ }^{|S A|}$ are also known.So,

$$
\frac{\overrightarrow{S a}}{|S a|}=\frac{\overrightarrow{S A}}{|S A|}
$$

From the formula above,the camera coordinate of point A can be obtained.

\section{The solution of pose[3].}

According to the previous theory, the coordinates of the flag points in the camera coordinate system can be obtained, whose coordinates in the target coordinates are known. As for the camera coordinate system $\mathrm{S}$ and target coordinate system $\mathrm{O}$, the coordinates of the number $i$ feature point in the two coordinate system are $\boldsymbol{S}_{i}=\left(x_{s i}, y_{s i}, z_{s i}\right)^{T}$ and ${ }^{\boldsymbol{O}_{i}=\left(x_{o i}, y_{o i}, z_{o i}\right)^{T}}$.According to conversion relationship between camera coordinates and world coordinates from the previous chapter, we can obtain:

$$
\boldsymbol{S}_{i}=\boldsymbol{R} \boldsymbol{O}_{i}+\boldsymbol{T}
$$

Nine linear equations can be obtained from correspondence between the three points. By the orthogonality constraint of $\boldsymbol{R}$, we can introduce three constraints, which make up linear equations with constraints. However, this solution requires iterative calculations, which is computationally intensive and difficult to guarantee accuracy.

Here we use a simple solution to find the rotation matrix $\boldsymbol{R}$. Then the amount of translation $\boldsymbol{T}$ can be obtained according to equation (11).The equation (11) is true not only for the corresponding points in two coordinate systems but also for the vectors. When the two vectors are both unit vectors, the corresponding relationship between the two vectors is rotation rather than translation, namely:

$$
\boldsymbol{n}_{s i}=\boldsymbol{R} \boldsymbol{n}_{o i}
$$


Among them, ${ }_{\text {si }}, \boldsymbol{n}_{\text {oi }}$ are two vectors in two coordinate systems of the same column vector $\boldsymbol{n}_{i}$. When there are three corresponding points, we can get linearly independent vectors by connecting any two points, which will be transformed into the unit vectors $\boldsymbol{n}_{1}, \boldsymbol{n}_{2}$.If $\boldsymbol{n}_{3}=\boldsymbol{n}_{1} \times \boldsymbol{n}_{2}$, the three vectors will be linearly independent. And the equation $\boldsymbol{n}_{s}=\boldsymbol{R} \boldsymbol{n}_{o}$ is true. Among them, $\boldsymbol{n}_{s}=\left[\begin{array}{lll}\boldsymbol{n}_{s 1} & \boldsymbol{n}_{s 2} & \boldsymbol{n}_{s 3}\end{array}\right]$, $\boldsymbol{n}_{o}=\left[\begin{array}{lll}\boldsymbol{n}_{01} & \boldsymbol{n}_{02} & \boldsymbol{n}_{03}\end{array}\right]$. The following equation can be obtained from the formula(8):

$$
\boldsymbol{R}=\boldsymbol{n}_{\mathrm{s}} \boldsymbol{n}_{o}^{-1}
$$

Then, we have $\boldsymbol{n}_{o 3}=\boldsymbol{n}_{o 1} \times \boldsymbol{n}_{o 2}$ and $\boldsymbol{n}_{s 3}=\boldsymbol{n}_{s 1} \times \boldsymbol{n}_{s 2}$, which can be taken into formula (13) to get $\boldsymbol{R}$. And $\boldsymbol{T}$ can be obtained from the formula (7).

\section{Model test}

We can get the rotation matrix by taking the experimental data into the method above:

\begin{tabular}{|c|c|c|}
\hline 0.954817 & -0.282335 & 0.066971 \\
\hline 0.235074 & 0.600867 & -0.762251 \\
\hline 0.174794 & 0.752350 & 0.640519 \\
\hline
\end{tabular}

Err $=0.192860$.

\section{Summary}

From the test, we can see that the error is very small. The method is feasible within the range of allowable error.

\section{References}

[1]. Yingming Hao,Feng Zhu,Jinjun Ou.Method of Three-dimensional Vision in Target Pose Measurement.China Image and Graphics.2002.p.1247 1249.

[2]. M.A.Fisher,R.C.Bolles.Random Sample Consensus:A Paradigm for Fitting with Application to Image Analysis and Automated Cartograghy. Communications of the ACM.1981.p.381 395.

[3]. Bingcheng Li,Jun Shen. Optimization of Rotation Matrix Estimation.Robot.1991.p.1 5. 